\title{
Synchrotron emission in molecular cloud cores: the SKA view
}

\author{
Marco Padovani and Daniele Galli
}

\begin{abstract}
INAF-Osservatorio Astrofisico di Arcetri, Largo E. Fermi 5, 50125 Firenze, Italy
\end{abstract}
e-mail: padovani@arcetri.astro.it, galli@arcetri.astro.it

Received 11 September 2018 / Accepted 20 November 2018

\begin{abstract}
Understanding the role of magnetic fields in star-forming regions is of fundamental importance. In the near future, the exceptional sensitivity of the Square Kilometre Array (SKA) will offer a unique opportunity to evaluate the magnetic field strength in molecular clouds and cloud cores through synchrotron emission observations. The most recent Voyager 1 data, together with Galactic synchrotron emission and Alpha Magnetic Spectrometer data, constrain the flux of interstellar cosmic-ray electrons between $\sim 3 \mathrm{MeV}$ and $\sim 832 \mathrm{GeV}$, in particular in the energy range relevant for synchrotron emission in molecular cloud cores at SKA frequencies. Synchrotron radiation is entirely due to primary cosmic-ray electrons, the relativistic flux of secondary leptons being completely negligible. We explore the capability of SKA in detecting synchrotron emission in two starless molecular cloud cores in the southern hemisphere, B68 and FeSt 1-457, and we find that it will be possible to reach signal-to-noise ratios of the order of 2-23 at the lowest frequencies observable by SKA $(60-218 \mathrm{MHz})$ with one hour of integration.
\end{abstract}

Key words. ISM: clouds - ISM: magnetic fields - cosmic rays

\section{Introduction}

During the last decades, many observational techniques have been developed to gather information on the magnetic field strength and geometry in molecular clouds and star forming regions such as Zeeman splitting of hyperfine molecular transitions (e.g. Crutcher et al. 1996), optical and near-infrared polarisation of starlight (e.g. Alves et al. 2008, 2011), polarisation of sub-millimetre thermal dust emission (e.g. Girart et al. 2009; Alves et al. 2018), maser emission polarisation (e.g. Vlemmings et al. 2011), Goldreich-Kylafis effect (Goldreich \& Kylafis 1981), and Faraday rotation (e.g. Wolleben \& Reich 2004). Together, all these techniques contribute to elucidating the still controversial role of magnetic fields in the process of star formation (e.g. Mouschovias et al. 1999; Mac Low \& Klessen 2004).

An additional method to probe magnetic fields in molecular clouds is via synchrotron radiation produced by relativistic electrons braked by a cloud's magnetic fields (e.g. Brown \& Marscher 1977). The intensity of the emission depends only on the electron density per unit energy and the projection of the magnetic field on the plane perpendicular to the line of sight. However, this technique is constrained by two limitations: (i) the poor knowledge of the interstellar (IS) flux of cosmic rays (CRs) below $\sim 500 \mathrm{MeV}$ and (ii) the limited sensitivity of current radio telescopes. Today, thanks to the latest data release of the Voyager 1 spacecraft (Cummings et al. 2016), the flux of $\mathrm{CR}$ electrons down to about $3 \mathrm{MeV}$ is well known. On the instrumental side, with the advent of the Square Kilometre Array (SKA), we are now approaching a new important era for highresolution observations at radio frequencies. As anticipated by Dickinson et al. (2015), in this paper we show that SKA will be able to detect synchrotron emission from molecular cloud cores and provide significant constraints on the magnetic field strength in molecular clouds.

It has been generally assumed that secondary leptons by primary $\mathrm{CR}$ protons are responsible for synchrotron emission in dense cores (e.g. Brown \& Marscher 1977; Jones et al. 2008), while primary CR electrons are generally neglected (for an exception see Dickinson et al. 2015). However, since $\mathrm{H}_{2}$ column densities in dense cores do not exceed $\sim 10^{23} \mathrm{~cm}^{-2}$, one can confidently argue that synchrotron emission is dominated instead by primary CR electrons. In fact, as shown by Padovani et al. (2018), the flux of secondary leptons at relativistic energies is negligible (see their Fig. 7). Secondary leptons dominate in the relativistic regime only at column densities larger than $\sim 10^{26} \mathrm{~cm}^{-2}$, that is, $\sim 500 \mathrm{~g} \mathrm{~cm}^{-2}$. At these very high column densities (typical of circumstellar discs rather than molecular clouds), the bulk of relativistic secondary leptons is created by the decay of charged pions and pair production from photons produced by Bremsstrahlung and neutral pion decay; see Padovani et al. (2018).

This paper is organised as follows: in Sect. 2 we recall the basic equations to compute the synchrotron specific emissivity; in Sect. 3 we report the most recent determinations of the IS CR electron flux; in Sect. 4 we describe the density and the magnetic field strength profiles that we use in Sect. 5 to compute the synchrotron flux density of starless molecular cloud cores; in Sect. 6 we discuss the implications for our results and summarise our most important findings.

\section{Basic equations}

In this section we present the main equations to compute the expected radio emission of a molecular cloud core, modelled as a 
spherically symmetric source. Unless otherwise indicated, in the following we use cgs units. The total power per unit frequency emitted by an electron of energy $E$ at frequency $v$ and radius $r$ is given by

$P_{v}^{\mathrm{em}}(E, r)=\frac{\sqrt{3} e^{3}}{m_{\mathrm{e}} c^{2}} B_{\perp}(r) F\left[\frac{v}{v_{c}\left(B_{\perp}, E\right)}\right]$

where $e$ is the elementary charge, $m_{\mathrm{e}}$ the electron mass, and $c$ the light speed (see e.g. Longair 2011). Here, $B_{\perp}(r)$ is the projection of the magnetic field on the plane perpendicular to the line of sight. The function $F$ is defined by

$F(x)=x \int_{x}^{\infty} K_{5 / 3}(\xi) \mathrm{d} \xi$,

where $K_{5 / 3}$ is the modified Bessel function of order $5 / 3$ and

$v_{c}\left(B_{\perp}, E\right)=\frac{3 e B_{\perp}}{4 \pi m_{\mathrm{e}} c}\left(\frac{E}{m_{\mathrm{e}} c^{2}}\right)^{2}=4.19\left(\frac{B_{\perp}}{\mathrm{G}}\right)\left(\frac{E}{m_{\mathrm{e}} c^{2}}\right)^{2} \mathrm{MHz}$

which is the frequency at which $F$ reaches its maximum value. The synchrotron specific emissivity $\epsilon_{v}(r)$ at frequency $v$ and radius $r$, integrated over polarisations and assuming an isotropic CR electron flux $j_{\mathrm{e}}(E, r)$ (see Sect. 3), namely the power per unit solid angle and frequency produced within unit volume, is

$\epsilon_{v}(r)=\int_{m_{\mathrm{e}} c^{2}}^{\infty} \frac{j_{\mathrm{e}}(E, r)}{v_{\mathrm{e}}(E)} P_{v}^{\mathrm{em}}(E, r) \mathrm{d} E$

where $v_{\mathrm{e}}(E)$ is the electron speed. The specific intensity is given by the specific emissivity integrated along a line of sight $s$. For a spherical source of radius $R$ (see Sect. 4.1),

$I_{\nu}(b)=\int_{0}^{s_{\max }(b)} \epsilon_{v}(s) \mathrm{d} s=2 \int_{b}^{R} \epsilon_{\nu}(r) \frac{r \mathrm{~d} r}{\sqrt{r^{2}-b^{2}}}$,

where $b$ is the impact parameter.

In principle, if the synchrotron radiation is sufficiently strong, synchrotron self-absorption can take place. In this case the emitting electrons absorb synchrotron photons and the emission is suppressed at low frequencies (see e.g. Rybicki \& Lightman 1986). We verified that the expected synchrotron emission of prestellar cores with typical magnetic field strengths of the order of $10 \mu \mathrm{G}-1 \mathrm{mG}$ is always optically thin even at the lowest frequencies reached by SKA. Additional suppression of synchrotron emission can be produced by the Tsytovich-Razin effect, arising when relativistic electrons are surrounded by a plasma (see e.g. Hornby \& Williams 1966; Munar-Adrover et al. 2013). This effect is negligible at frequencies $v \gg v_{\mathrm{TR}}=20\left(n_{\mathrm{e}} / \mathrm{cm}^{-3}\right)(B / \mathrm{G})^{-1}$, where $n_{\mathrm{e}}$ is the electron volume density. Even maximising $v_{\mathrm{TR}}$ by assuming a magnetic field strength of $10 \mu \mathrm{G}$, a total volume density of $10^{6} \mathrm{~cm}^{-3}$, and a typical ionisation fraction of $10^{-7}, v_{\mathrm{TR}}=200 \mathrm{kHz}$. Therefore, we can safely neglect this effect since $v_{\mathrm{TR}}$ is below the frequency range of SKA1-Low $(60-302 \mathrm{MHz})$ and SKA1-Mid $(0.41-12.53 \mathrm{GHz})$ according to the most recent specifications ${ }^{1}$.

1 https://astronomers . skatelescope.org/wp-content/ uploads/2017/10/SKA-TEL-SKO-0000818-01_SKA1_Science_ Perform.pdf.

\section{Interstellar cosmic-ray electrons}

The CR electron flux at energies below $\sim 1 \mathrm{GeV}$ can be subject to propagation effects upon their penetration into cores due to magnetohydrodynamical turbulence generated by CR protons, causing modulation at low energies (Ivlev et al. 2018). While the consequences of this process will be addressed in a following study (Ivlev et al., priv. comm.), in this paper we assume the molecular cloud cores to be exposed to the IS CR electron flux $j_{\mathrm{e}}^{\mathrm{IS}}$. The latter is now well constrained between about $500 \mathrm{MeV}$ and $20 \mathrm{GeV}$ by Galactic synchrotron emission (Strong et al. 2011; Orlando 2018) and at lower energies $(3 \mathrm{MeV} \lesssim E \lesssim 40 \mathrm{MeV}$ ) by the recent Voyager 1 observations (Cummings et al. 2016). The latest Voyager data release shows that the electron flux increases with decreasing energy as $E^{-p}$ with a slope $p=1.3$. At energies above a few gigaelectron volts, the slope is constrained by Alpha Magnetic Spectrometer data (hereafter AMS-02; Aguilar et al. 2014), and is $p=3.2$. Ivlev et al. (2015) and Padovani et al. (2018) combined the lowand high-energy trends with the fitting formula

$j_{\mathrm{e}}^{\mathrm{IS}}(E)=j_{0} \frac{E^{a}}{\left(E+E_{0}\right)^{b}}$,

where $j_{0}=2.1 \times 10^{18} \mathrm{eV}^{-1} \mathrm{~s}^{-1} \mathrm{~cm}^{-2} \mathrm{sr}^{-1}, a=-1.3, b=1.9$, $E_{0}=710 \mathrm{MeV}$, and $E$ is in electron volts. Most of the synchrotron radiation is emitted around a frequency $v=0.29 v_{c}$ (Longair 2011), corresponding to electrons of energy

$E_{\mathrm{syn}} \approx 464\left(\frac{v}{\mathrm{~Hz}}\right)^{1 / 2}\left(\frac{B}{\mathrm{G}}\right)^{-1 / 2} \mathrm{eV}$.

For typical values of the magnetic field strength measured in starless cores $(10 \mu \mathrm{G}-1 \mathrm{mG})$, the corresponding values of $E_{\text {syn }}$ for the frequency range of SKA (see Sect. 2) span the energy interval from $\sim 100 \mathrm{MeV}$ to $\sim 20 \mathrm{GeV}$ as shown in Fig. 1. For cloud cores with relatively strong magnetic fields (e.g. $\sim 500 \mu \mathrm{G})$ observed at $v \lesssim 600 \mathrm{MHz}, E_{\text {syn }}$ is lower than $\sim 500 \mathrm{MeV}$. In this energy range Voyager data provide a better constraint on the CR electron flux than Galactic synchrotron emission and AMS-02 data.

\section{Density and magnetic field profiles}

\subsection{Density profile}

A common feature of starless cores derived from millimetre(mm)-continuum observations is a central flattening in the density profile, with typical maximum densities of the order of $10^{5}-10^{6} \mathrm{~cm}^{-3}$, reaching $\sim 2 \times 10^{7} \mathrm{~cm}^{-3}$ in the starless core L1544 (Keto \& Caselli 2010). Various density profiles are used to reproduce observations of dense cores such as the Bonnor-Ebert sphere or softened power laws. In the following we adopt the latter profile as given by Tafalla et al. (2002)

$n(r)=\frac{n_{0}}{1+\left(r / r_{0}\right)^{q}}$,

where $n_{0}$ is the central density, $r_{0}$ the radius of the central flat region, and $q$ the asymptotic slope.

Since the specific emissivity is a function of radius (Eq. (4)), in principle one should consider the attenuation of the IS CR electron flux due to energy losses (Padovani et al. 2009). However, the typical maximum $\mathrm{H}_{2}$ column densities of starless cores are of the order of $10^{23} \mathrm{~cm}^{-2}$; therefore only electrons 


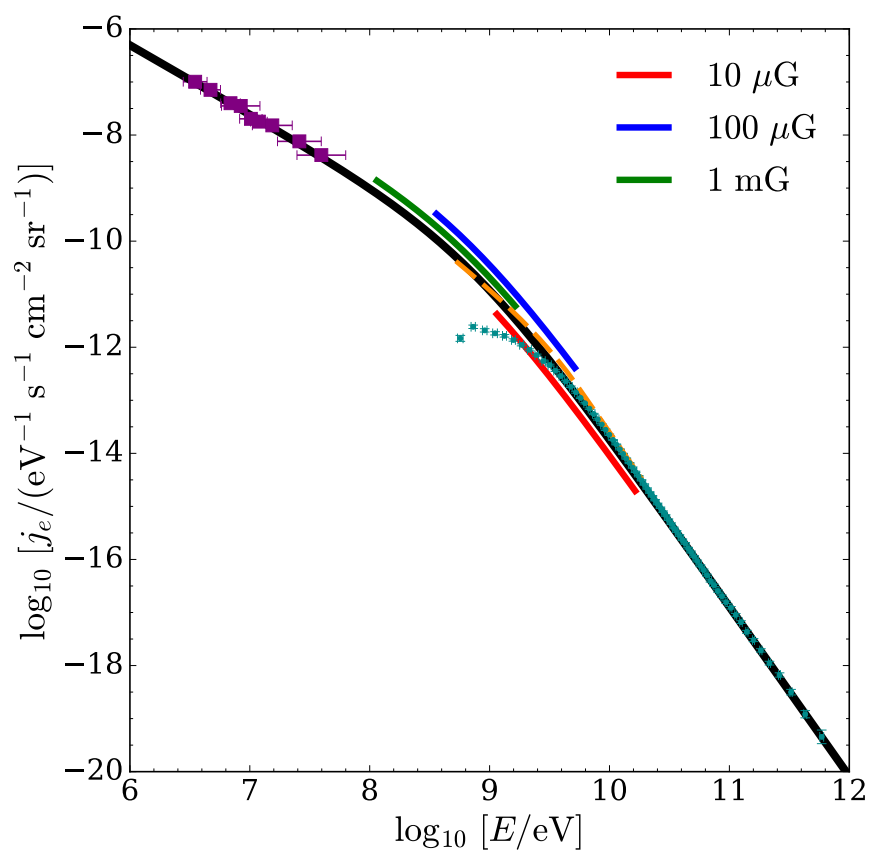

Fig. 1. Flux of IS CR electrons (black solid line) as a function of energy. Data: Voyager 1 (Cummings et al. 2016, solid purple squares) at low energies, AMS-02 (Aguilar et al. 2014, solid cyan circles) at high energies. The red, blue, and green thick lines show the energy range that mostly contributes to synchrotron emission in the frequency range of SKA for the values of the magnetic field strength listed in the legend (see Eq. (7)). The orange dashed line shows the CR electron-positron flux obtained from Galactic synchrotron emission (Orlando 2018).

with energies lower than $1 \mathrm{MeV}$ can be stopped completely (Padovani et al. 2018). One should also take into account the fact that CRs follow helical trajectories along magnetic field lines, meaning that the effective column density passed through is larger than the corresponding line-of-sight column density (see e.g. Padovani et al. 2013). Strong bending of field lines is not expected in starless cores: assuming a poloidal field configuration, the effective column density is larger at most by a factor of approximately three (see Fig. 3 in Padovani \& Galli 2011). We can then assume the effective column density and the line-ofsight column density to be the same. Besides, the minimum electron energy contributing to synchrotron emission for the range of frequencies and magnetic field strengths considered here is $\sim 100 \mathrm{MeV}$, corresponding to a value of the stopping range of the order of $6 \times 10^{24} \mathrm{~cm}^{-2}$. Therefore, in Eq. (4), we can safely neglect the dependence of the CR electron flux on column density (i.e. on radius $r$ ).

\subsection{Magnetic field strength profile}

For the magnetic field strength we assume the relation

$B(n)=B_{0}\left(\frac{n}{n_{0}}\right)^{\kappa}$,

where $B_{0}$ is the value of the magnetic field strength at the central density $n_{0}$ and $\kappa \sim 0.5-0.7$ is determined by observations (see e.g. Crutcher 2012). We use Eq. (9) as a local relation to obtain the strength of the magnetic field from the radial profile of $n$. While the choice of the density profile is unimportant for the attenuation of the CR electron flux (see Sect. 4.1), it has important consequences on the profile of $B$ and, in turn, on the

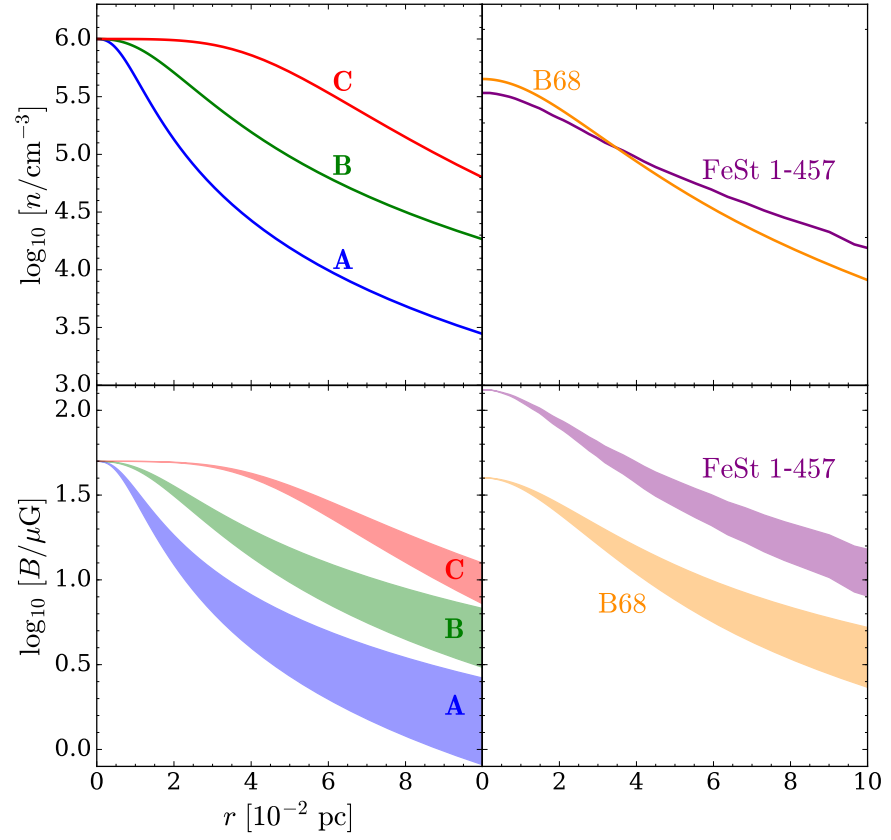

Fig. 2. Density and magnetic field strength profiles as a function of radius (upper and lower panels, respectively). Left column: models of starless cores described by Eq. (8), $r_{0}=14^{\prime \prime}, q=2.5$ (model A, solid blue line), $r_{0}=30^{\prime \prime}, q=2.5$ (model $\mathrm{B}$, solid green line), and $r_{0}=75^{\prime \prime}, q=4$ (model C, solid red line). Right column: starless cores FeSt 1-457 (purple) and B68 (orange). Shaded areas in the lower panels encompass the curves obtained with Eq. (9) using $\kappa=0.5$ and 0.7 for models A, B, C and B68 and $\kappa=0.68$ and 0.88 for FeSt 1-457 (upper and lower boundary, respectively).

observed flux density. In the following section we compute the flux densities for different assumptions on the magnetic field profile. Synchrotron emissivity depends on $B_{\perp}$ (see Eq. (4)). Here we simply assume $B_{\perp}=\pi B / 4$ to account for a random orientation of the magnetic field with respect to the line of sight.

\section{Modelling}

\subsection{General case}

We consider three models of starless cores with outer radius $R=$ $0.1 \mathrm{pc}$ and central density $n_{0}=10^{6} \mathrm{~cm}^{-3}$. We vary the radius of the central flat region and the asymptotic slope (the parameters $r_{0}$ and $q$, respectively, in Eq. (8)) ranging from a relatively peaked profile ( $r_{0}=14^{\prime \prime}, q=2.5$, model A) to an almost uniform profile $\left(r_{0}=75^{\prime \prime}, q=4\right.$, model C), including an intermediate case $\left(r_{0}=\right.$ $30^{\prime \prime}, q=2.5$, model B). These profiles are similar to those used by Tafalla et al. (2002) to describe low-mass starless cores in the Taurus molecular cloud. The magnetic field strength is assumed equal to $B_{0}=50 \mu \mathrm{G}$ at the centre of the core in all models, and we consider the two cases $\kappa=0.5$ and 0.7 (Crutcher 2012). The left column of Fig. 2 shows both the density and magnetic field strength profiles of our models.

We compute the specific intensity from Eqs. (4) and (5), assuming the CR electron flux given by Eq. (6). The observable quantity is the flux density $S_{v}$, obtained by integrating over the solid angle the specific intensity multiplied by the telescope pattern. The latter is assumed to be a Gaussian with beam full width at half maximum $\theta_{b}=300^{\prime \prime}$ equal to the angular size of our starless core models at a distance of $140 \mathrm{pc}$ (non-resolved source). 


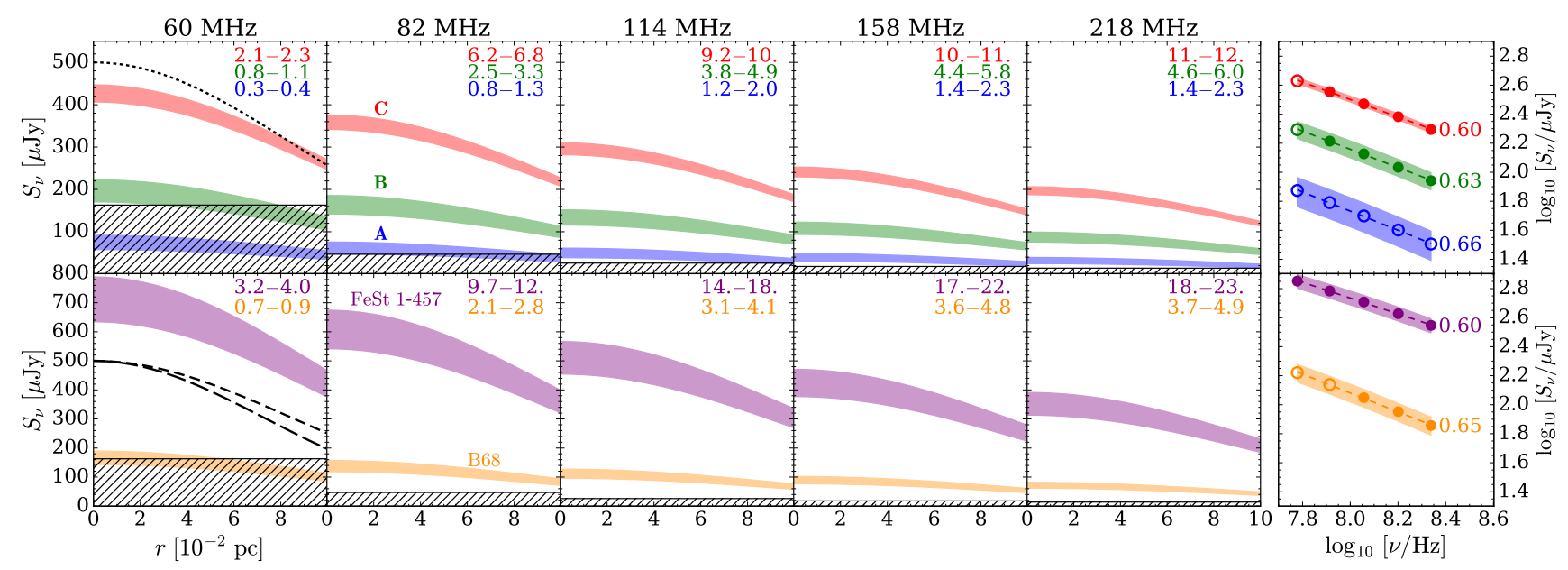

Fig. 3. Radial flux density profiles for the starless core models described in Sect. 5.1 (upper row) and for B68 and FeSt 1-457 (lower row, see Sect. 5.2). The observing frequency is shown in black at the top of each column, while numbers in the upper-right corner of each subplot represent the radius-averaged $\mathrm{S} / \mathrm{N}$ for the two values of $\kappa(0.5$ and 0.7 for models $\mathrm{A}, \mathrm{B}, \mathrm{C}$ and B68, and 0.68 and 0.88 for FeSt 1-457, see Eq. (9)). Shaded areas encompass the curves obtained with Eq. (9) by using the two values of $\kappa$ (see Fig. 2 for colour-coding). The telescope beam is shown in the leftmost column for models A, B, and C (dotted black line, 300"), B68 (short-dashed black line, 330"), and FeSt 1-457 (long-dashed black line, $\left.284^{\prime \prime}\right)$. Hatched areas display SKA sensitivities for one hour of integration at different frequencies. The two panels on the right side show the flux density as a function of frequency. Empty (solid) circles refer to a S/N smaller (larger) than 3, respectively. The spectral index $\alpha$ is shown on the right of each curve.

The upper row of Fig. 3 displays the flux density profiles at the five lowest frequencies of SKA1-Low for the three models and the sensitivity limits for one hour of integration. At higher frequencies, a source of $300^{\prime \prime}$ is resolved, with consequent loss of flux. However, the total flux density can still be recovered by convolution with a larger beam.

It is evident that what determines the maximum value of $S_{v}$ is not only the maximum magnetic field strength, but also the integrated value of $B$ along the line of sight, which, in turn, depends on the density profile: the shallower the density profile, the higher $S_{v}$ is. As a consequence, for one hour of integration, a centrally peaked starless core such as model A is detectable with a signal-to-noise ratio $(\mathrm{S} / \mathrm{N})$ of about 2 only at frequencies higher than $158 \mathrm{MHz}$, whereas a less centrally concentrated core such as model B will be observable with $S / N \gtrsim 3$ at $v \gtrsim 114 \mathrm{MHz}$. For an almost uniform density profile such as model C, a $S / N \gtrsim 2$ is promptly reached at $v \gtrsim 60 \mathrm{MHz}$.

If the CR electron flux is proportional to $E^{-p}$, the synchrotron flux density varies as $S_{\nu} \propto v^{-\alpha}$, where $\alpha=(p-1) / 2$ is the spectral index (Rybicki \& Lightman 1986). For our assumed CR electron flux, $p \rightarrow 1.3$ at low energies and $p \rightarrow 3.2$ at high energies (see Sect. 3), therefore $\alpha$ is expected to be intermediate between 0.2 and 1.1. In fact, our calculations show that $\alpha$ is about 0.6 in the frequency range $60-218 \mathrm{MHz}$ (see the two rightmost panels in Fig. 3). Since in starless cores the IS CR electron flux is not attenuated, we expect the spectral index $\alpha \sim 0.6$ to be weakly dependent on density and magnetic field strength profiles at SKA1-Low frequencies as long as $10 \mu \mathrm{G} \lesssim B \lesssim 1 \mathrm{mG}$.

The flux density depends weakly on the value of $\kappa$ (from $10 \%$ in model $\mathrm{C}$ to $35 \%$ in model $\mathrm{A}$, for $\kappa$ ranging from 0.5 to 0.7 ), but it is strongly controlled by the strength of the magnetic field (Eq. (9)). For a power-law electron flux proportional to $E^{-p}$ the synchrotron emissivity is $\epsilon_{\nu} \propto B^{\delta}$, where $\delta=(p+1) / 2$ (see e.g. Rybicki \& Lightman 1986). Since $p$ varies between 1.3 and 3.2, we expect $S_{v}$ to increase with the magnetic field strength with a dependence intermediate between $\delta=1.2$ and 2.1. Increasing the value of $B_{0}$ from $10 \mu \mathrm{G}$ to $1 \mathrm{mG}$ in the case of the core model B we obtain the results shown in Fig. 4 (models $\mathrm{A}$ and $\mathrm{C}$ give similar results). In the frequency range $60 \mathrm{MHz}-218 \mathrm{MHz}$ the flux density increases roughly in a power-law fashion with exponent $\sim 1.5-1.6$. As a consequence, a larger value of $B_{0}$ would make our modelled sources detectable with a shorter integration time.

Finally, we note that in this work we consider typical outer radii of the order of $0.1 \mathrm{pc}$, but for bigger starless cores - especially with flat density profile - the flux density would be correspondingly larger than that shown in Fig. 3.

\subsection{Magnetic field strength in FeSt 1-457 and B68}

In this section we show the potential of SKA in observing two starless cores in the southern hemisphere: FeSt 1-457 (also known as Core 109 in the Pipe Nebula), and B68 in Ophiuchus. The density profiles of FeSt 1-457 and B68 are taken from Juárez et al. (2017) and Galli et al. (2002), respectively. According to Kandori et al. (2018), the magnetic field at the centre of FeSt $1-457$ is $B_{0} \sim 132 \mu \mathrm{G}$ decreasing with a slope $\kappa=$ $0.78 \pm 0.10$, while the plane-of-sky magnetic field strength in B68 has been estimated by Kandori et al. (2009) as $\sim 20 \mu \mathrm{G}$. Since the total strength for a random field is about twice that measured along any direction (Shu et al. 1999), in the following we consider $B_{0}=40 \mu \mathrm{G}$ for B68 in Eq. (9). The right column of Fig. 2 shows the density and the magnetic field strength profiles for these two starless cores.

Similarly to the three modelled cores in Sect. 5.1, we compute the flux density using a beam size equal to the angular size of B68 and FeSt 1-457 (330" and 284", respectively ${ }^{2}$ ). The results are shown in the lower row of Fig. 3. We find that a $\mathrm{S} / \mathrm{N}$ larger than $\sim 2$ is reached in one hour of integration at $v \gtrsim 82 \mathrm{MHz}$ for B68, while FeSt 1-457 can be detected even at the lowest frequency $v=60 \mathrm{MHz}$ with $S / N \gtrsim 3$.

2 We assume a radius $R=0.1 \mathrm{pc}$ for both cores and a distance of $125 \mathrm{pc}$ for B68 (de Geus et al. 1989) and $145 \mathrm{pc}$ for FeSt 1-457 (Alves \& Franco 2007). 


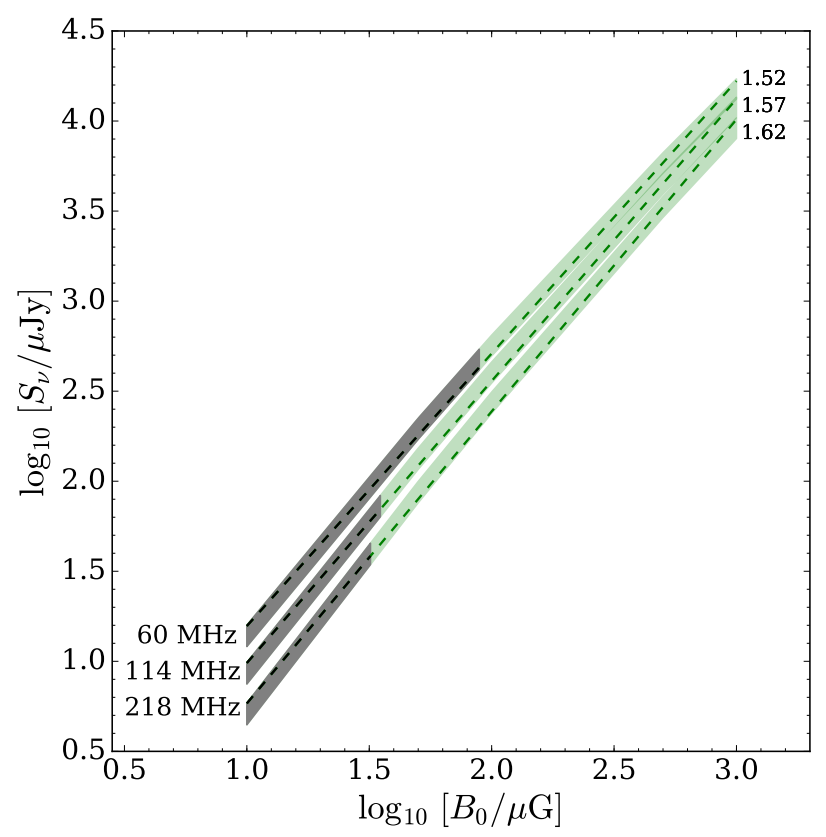

Fig. 4. Flux density at 60,114 , and $218 \mathrm{MHz}$ (lower-left labels) as a function of magnetic field strength $B_{0}$ for model B (see Sect. 5.1). Green shaded areas encompass the curves obtained with Eq. (9) by using $\kappa=$ 0.5 and 0.7. Grey areas correspond to $S / N<3$ and dashed lines are power-law fits of $S_{v} \propto B_{0}^{\delta}$ with $\delta$ listed in the upper-right corner.

\section{Conclusions}

We explored the capability of SKA in detecting synchrotron emission from starless cores. This observing facility is very promising because it can provide robust constraints on the magnetic field strength, thus contributing to elucidate the role of magnetic fields in the star-formation process. Our main conclusions are as follows.

Thanks to the recent Voyager 1 measurements, combined with AMS-02 data and Galactic synchrotron emission, the flux of IS CR electrons is now well-known down to $3 \mathrm{MeV}$. Synchrotron emission is entirely dominated by primary CR electrons, while the contribution by secondary relativistic leptons is negligible.

For typical values of the magnetic field strength in starless cores $(\sim 10 \mu \mathrm{G}-1 \mathrm{mG})$, synchrotron emission in the SKA1-Low and SKA1-Mid frequency bands is determined by electrons with energies between $\sim 100 \mathrm{MeV}$ and $\sim 20 \mathrm{GeV}$. The stopping range at $\sim 100 \mathrm{MeV}$ is of the order of $6 \times 10^{24} \mathrm{~cm}^{-2}$, therefore the IS CR electron flux contributing to synchrotron emission is attenuated at $\mathrm{H}_{2}$ column densities much in excess of the typical column densities of starless cores.

The synchrotron radiation emitted by a starless core depends on the integrated line-of-sight value of the magnetic field strength, which, in turn, depends on the density profile. As a result, for the same maximum value of the magnetic field strength, a starless core with a shallow density profile shows a larger synchrotron flux density than a core with a peaked profile.

We modelled two starless cores in the southern hemisphere whose density profiles and typical magnetic field strengths are well-constrained by observations (B68 and FeSt 1-457) and we found that SKA will be able to detect synchrotron emission at $82 \mathrm{MHz} \lesssim v \lesssim 218 \mathrm{MHz}$ reaching a $S / N \sim 2-5$ and $\sim 10-23$ in one hour of integration for B68 and FeSt 1-457, respectively; FeSt 1-457 can be detected even at the lowest frequency $v=$ $60 \mathrm{MHz}$ with a $\mathrm{S} / \mathrm{N} \gtrsim 3$.
We found a typical spectral index $\alpha=(p-1) / 2 \sim 0.6$ intermediate between the values 0.2 and 1.1 resulting from the lowand high-energy asymptotic slopes of the primary CR electron flux ( $p \rightarrow 1.3$ and $p \rightarrow 3.2$, respectively). Since in starless cores the IS CR electron flux is not attenuated by losses (although it can be modulated by self-generated turbulence; see Ivlev et al. 2018), we expect $\alpha \sim 0.6$ to be weakly dependent on density and magnetic field strength profiles at SKA1-Low frequencies.

Our study shows that SKA is a powerful instrument to constrain the strength of magnetic fields in molecular cloud cores. This facility and the proposed next-generation Very Large Array (ngVLA, Murphy et al. 2018) have the capability to shed light in the near future on the role of magnetic fields in the process of star formation.

Acknowledgements. We want to thank the referee for her/his helpful comments, which further improved the quality of this paper. MP acknowledges funding from the European Unions Horizon 2020 research and innovation programme under the Marie Skłodowska-Curie grant agreement No 664931. The authors thank Maite Beltrán, Riccardo Cesaroni, and Francesco Fontani for valuable discussions and the referee for useful suggestions and comments.

\section{References}

Alves, F. O., \& Franco, G. A. P. 2007, A\&A, 470, 597

Alves, F. O., Franco, G. A. P., \& Girart, J. M. 2008, A\&A, 486, L13

Alves, F. O., Acosta-Pulido, J. A., Girart, J. M., Franco, G. A. P., \& López, R. 2011, AJ, 142, 33

Alves, F. O., Girart, J. M., Padovani, M., et al. 2018, A\&A, 616, A56

Aguilar, M., Aisa, D., Alvino, A., et al. 2014, Phys. Rev. Lett., 113, 121102

Brown, R. L., \& Marscher, A. P. 1977, ApJ, 212, 659

Crutcher, R. M. 2012, ARA\&A, 50, 29

Crutcher, R. M., Troland, T. H., Lazareff, B., \& Kazes, I. 1996, ApJ, 456, 217

Cummings, A. C., Stone, E. C., Heikkila, B. C., et al. 2016, ApJ, 831, 18

de Geus, E. J., de Zeeuw, P. T., \& Lub, J. 1989, A\&A, 216, 44

Dickinson, C., Beck, R., Crocker, R., et al. 2015, Advancing Astrophysics withthe Square Kilometre Array (AASKA14), 102

Galli, D., Walmsley, M., \& Gonçalves, J. 2002, A\&A, 394, 275

Girart, J. M., Beltrán, M. T., Zhang, Q., Rao, R., \& Estalella, R. 2009, Science, 324, 1408

Goldreich, P., \& Kylafis, N. D. 1981, ApJ, 243, L75

Hornby, J. M., \& Williams, P. J. S. 1966, MNRAS, 131, 237

Ivlev, A. V., Padovani, M., Galli, D., \& Caselli, P. 2015, ApJ, 812, 135

Ivlev, A. V., Dogiel, V. A., Chernyshov, D. O., et al. 2018, ApJ, 855, 23

Jones, D. I., Protheroe, R. J., \& Crocker, R. M. 2008, PASA, 25, 161

Juárez, C., Girart, J. M., Frau, P., et al. 2017, A\&A, 597, A74

Kandori, R., Tamura, M., Tatematsu, K.-I., et al. 2009, IAU Symp., 259, 107

Kandori, R., Tomisaka, K., Tamura, M., et al. 2018, ApJ, 865, 121

Keto, E., \& Caselli, P. 2010, MNRAS, 402, 1625

Longair, M. S. 2011, High Energy Astrophysics (Cambridge: Cambridge University Press)

Mac Low, M.-M., \& Klessen, R. S. 2004, Rev. Mod. Phys., 76, 125

Mouschovias, T. C., \& Ciolek, G. E. 1999, in NATO Advanced Science Institutes(ASI) Ser. C, eds. C. J. Lada, \& N. D. Kylafis, 540, 305

Munar-Adrover, P., Bosch-Ramon, V., Paredes, J. M., \& Iwasawa, K. 2013, A\&A, 559, A13

Murphy, E. J., Bolatto, A., Chatterjee, S., et al. 2018, ArXiv e-prints [arXiv: 1810.07524$]$

Orlando, E. 2018, MNRAS, 475, 2724

Padovani, M., \& Galli, D. 2011, A\&A, 530, A109

Padovani, M., Galli, D., \& Glassgold, A. E. 2009, A\&A, 501, 619

Padovani, M., Hennebelle, P., \& Galli, D. 2013, A\&A, 560, A114

Padovani, M., Ivlev, A. V., Galli, D., \& Caselli, P. 2018, A\&A, 614, A111

Rybicki, G. B., \& Lightman, A. P. 1986, Radiative Processes in Astrophysics (New Jersey: John Wiley \& Sons), 400

Shu, F. H., Allen, A., Shang, H., Ostriker, E. C., \& Li, Z.-Y. 1999, ed. C. J. Lada \& N. D. Kylafis, 193

Strong, A. W., Orlando, E., \& Jaffe, T. R. 2011, A\&A, 534, A54

Tafalla, M., Myers, P. C., Caselli, P., Walmsley, C. M., \& Comito, C. 2002, ApJ, 569,815

Vlemmings, W. H. T., Humphreys, E. M. L., \& Franco-Hernández, R. 2011, ApJ, 728,149

Wolleben, M., \& Reich, W. 2004, A\&A, 427, 537 